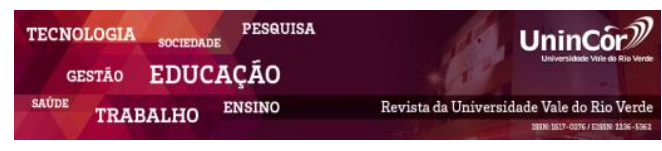

Revista da Universidade Vale do Rio Verde ISSN: 1517-0276 / EISSN: 2236-5362 Vol. 16 | n. 1 | Ano 2018

Júnia Rafael Mendonça Figueiredo Universidade Vale do Rio Verde (UninCor) prof.junia.figueiredo@unincor.edu.br

Patrícia Duarte de Oliveira Paiva Universidade Federal de Lavras (UFLA) patriciapaiva@dag.ufla.br

Renato Paiva

Universidade Federal de Lavras (UFLA) renpaiva@dbi.ufla.br

Diogo Pedrosa Corrêa da Silva Universidade Federal de Lavras (UFLA) pedrosacorrea@hotmail.com

Raquel Mesquita

Universidade Federal de Lavras (UFLA) raquel.mesquita@yahoo.com.br

Camila Vitória Nunes de Faria Universidade Federal de Lavras (UFLA) camila-vitoria@hotmail.com

Michele Valquíria dos Reis Universidade Federal de Lavras (UFLA) mvreis@yahoo.com.br

\section{CONSERVAÇÃO DE Genipa americana: TÉCNICAS DE ENCAPSULAMENTO E CRESCIMENTO LENTO}

\section{RESUMO}

Segmentos nodais de Genipa americana foram armazenados utilizando técnicas de encapsulamento e crescimento lento. Para o armazenamento de unidades encapsuláveis foram avaliados a influência da matriz de alginato de sódio a $3 \%$ e $4 \%$, o efeito de um pré-tratamento com soluções de sacarose a $0,0,25$ e $0,50 \mathrm{M}$, bem como o efeito da temperatura de $8^{\circ} \mathrm{C}$ e $15^{\circ} \mathrm{C}$ durante 30 dias de armazenamento, sendo avaliada a porcentagem de ruptura dessas cápsulas após 50 dias de inoculação. Para o crescimento lento foram avaliadas diferentes concentrações de sacarose (30, 45 e $60 \mathrm{~g} \mathrm{~L}^{-1}$ ) e temperatura $8^{\circ} \mathrm{C}$ e $15^{\circ} \mathrm{C}$ após 90 e 180 dias de armazenamento. Sendo avaliada a porcentagem de sobrevivência e regeneração, comprimento da parte área, número de brotos e folhas. Apenas as unidades encapsuláveis armazenadas à $15^{\circ} \mathrm{C}$ sobreviveram. Nesta temperatura, a ruptura da cápsula foi melhor no pré-tratamento com $0 \mathrm{M}$ de sacarose. Para o crescimento lento, a temperatura de $8^{\circ}$ também não permitiu sobrevivência dos explantes. Assim, após 180 dias os melhores resultados ocorreram na temperatura de $15^{\circ} \mathrm{C}$ com acréscimo de $60 \mathrm{~g} \mathrm{~L}^{-1}$ de sacarose ao meio de cultura. Dessa forma, pode-se concluir que é possível fazer o armazenamento da espécie utilizando essas técnicas.

Palavras-chave: Jenipapo. Conservação in vitro. Unidades encapsuláveis. Temperatura. Cerrado.

\section{CONSERVATION OF Genipa americana: ENCAPSULATION AND SLOW GROWTH TECHNIQUES}

\section{RESUMO}

Nodal segments of Genipa americana were stored using encapsulation and slow growth techniques. The influence of the $3 \%$ and $4 \%$ sodium alginate matrix, the effect of a pretreatment with $0,0,25$ and $0,50 \mathrm{M}$ sucrose solutions, as well the effect of $8^{\circ} \mathrm{C}$ and $15^{\circ} \mathrm{C}$ temperature during storege for 30 days. It was evaluated the percentage of rupture of these capsules after 50 days of inoculation. For the slow growth, different sucrose concentrations $\left(30,45\right.$ and $\left.60 \mathrm{~g} \mathrm{~L}^{-1}\right)$ and temperature $8^{\circ} \mathrm{C}$ and $15^{\circ} \mathrm{C}$ after storege for 90 and 180 days were evaluated. It was evaluated the percentage of survival and regeneration, shoot lenght, shoots and leaves number. Only the encapsulating units stored at $15{ }^{\circ} \mathrm{C}$ survived. At this temperature, the capsule rupture was better in pretreatment with $0 \mathrm{M}$ sucrose. For the slow growth, the temperature of $8^{\circ} \mathrm{C}$ also did not allow the survival of the explants. Thus, after 180 days the best results occurred at the temperature of $15{ }^{\circ} \mathrm{C}$ with the addition of $60 \mathrm{~g} \mathrm{~L}^{-1}$ of sucrose to the culture medium. In this way, it can be concluded that it is possible to make the storage of the species using these techniques.

Palavras-chave: Jenipapo. In vitro conservation. Encapsulating units. Temperature. Cerrado. 


\section{INTRODUÇÃO}

O Brasil é formado por seis tipos de biomas continentais (Amazônia, Cerrado, Mata Atlântica, Caatinga, Pampa e Pantanal). Dentre esses biomas, o Cerrado é o segundo maior em extensão, apresentando uma área de 2.036 .448 $\mathrm{km}^{2}$, o que corresponde a $23,9 \%$ do território brasileiro (BRASIL, 2004). Esse bioma apresenta mais de 4.800 espécies de plantas e vertebrados, sendo um dos principais hotspots, com uma taxa de desmatamento 2,5 vezes maior do que a Amazônia, o que coloca muitas espécies em risco de extinção (STRASSBURG et al., 2017).

Dessa forma, diversos estudos têm sido realizados no intuito de aumentar a produção de mudas de espécies nativas, as quais podem ser utilizadas para o reflorestamento de áreas degradadas (FERNANDES et al., 2000). Dentre as espécies que estão sendo estudadas, destaca-se Genipa americana (jenipapeiro), uma espécie frutífera, com importância florestal e com propriedades fitorremediadoras (ALMEIDA et al., 2015; LÉDO et al., 2015; SANTANA et al., 2012). No entanto, existem problemas relacionados com a propagação e conservação do jenipapo, uma vez que, suas sementes apresentam germinação lenta e desuniforme, bem como rápida perda de viabilidade, o que limita a sua inclusão em bancos de germoplasma e a conservação da espécie por sementes (LÉDO et al., 2015; SÁ et al., 2015; SOUZA et al., 2016;).
Uma alternativa para a conservação de espécies que apresentam problemas com a propagação e conservação de sementes é a utilização do encapsulamento de embriões somáticos, segmentos nodais e ápices caulinares, os quais posteriormente podem ser armazenados por um curto período de tempo (DAUD; TAHA; HASBULLAH, 2008). No entanto, a composição adequada da cápsula utilizada durante a técnica e também condições ambientais ótimas de armazenamento são necessárias para que a conservação ocorra de forma satisfatória (RAI , JAISWAL, JAISWAL, 2008; SHARMA et al. 2013).

Além dessa técnica, a conservação in vitro, como o crescimento lento, também surge como alternativa viável para a conservação dessas espécies. Com essa técnica é possível fazer a conservação segura, barata e em médio prazo do material vegetal, sendo esse mantido em condições assépticas por vários meses, sem ter a necessidade de serem realizados subcultivos periódicos (LAMBARDI e OZUDOGRU, 2012). Para que isso seja possível, são realizadas alterações nos meios de cultura e também nas condições ambientais, o que consequentemente causa redução do metabolismo vegetal, proporcionando a sua conservação (ENGELMANN, 2011; OZUDOGRU, PREVIATI, LAMBARDI, 2010; SILVA et al., 2018). 
Dessa forma, objetivou-se fazer a conservação de jenipapo, utilizando técnicas de armazenamento de unidades encapsuláveis e crescimento lento.

\section{MATERIAL E MÉTODOS}

\subsection{Obtenção do material vegetal}

Frutos maduros de jenipapeiro (Genipa americana) foram coletados em latitude $21^{\circ}$ $13^{\prime} 40$ " S e longitude $44^{\circ} 57^{\prime} 50^{\circ \prime} \mathrm{W}$, a uma altitude de $919 \mathrm{~m}$. Os frutos foram despolpados e as sementes foram retiradas e lavadas em água corrente, retirando-se completamente à mucilagem, e então acondicionadas em papel toalha à temperatura ambiente por 24 horas para secagem. Posteriormente, passaram por um processo de embebição por 48 horas e foram inoculadas em meio MS com a metade das concentrações dos sais minerais (MURASHIGE e SKOOG, 1962), acrescido de $1,5 \mathrm{~g} \mathrm{~L}^{-1}$ de sacarose e $7 \mathrm{~g} \mathrm{~L}^{-1}$ de ágar, com $\mathrm{pH}$ ajustado para 5,8 e autoclavado à $121^{\circ} \mathrm{C}$ por 20 minutos. Após a inoculação, o material foi mantido no escuro por 16 dias e então transferido para sala de crescimento com fotoperíodo de 16 horas, temperatura de $25 \pm 2^{\circ} \mathrm{C}$ com irradiância de fótons de $36 \mu \mathrm{mol} \mathrm{m}^{-2} \mathrm{~s}^{-1}$ (SOUZA et al., 2016).

\subsection{Armazenamento de unidades encapsuláveis}

Segmentos nodais com aproximadamente $2 \mathrm{~mm}$ foram excisados de plântulas obtidas a partir da germinação das sementes descritas no item anterior. Esses explantes foram adicionados às diferentes soluções de alginato de sódio (Sigma-Aldrich), obtidas a partir da diluição de 3\% (SÁ et al., 2015) e 4\% desse composto em meio MS. Em seguida, com o auxílio de pipeta automática e ponteiras autoclavadas, os segmentos nodais foram resgatados individualmente e gotejados em solução de cloreto de cálcio $(100 \mathrm{mM})$ na qual permaneceram por 20 minutos (período de complexação). Após esse período, as cápsulas foram imersas em água destilada e autoclavada para retirada do excesso de cloreto de cálcio e submetidas a soluções de $0,0,25$ e $0,50 \mathrm{M}$ de sacarose, onde permaneceram 16 horas em agitação. Em seguida, algumas cápsulas passaram pelo processo de descomplexação em solução de nitrato de potássio $(100 \mathrm{mM})$ por 15 minutos e foram inoculadas em meio de regeneração (MS meia força $+1,5 \mathrm{~g} \mathrm{~L}^{-1}$ de sacarose $+7 \mathrm{~g} \mathrm{~L}^{-1}$ de ágar, com $\mathrm{pH}$ ajustado para 5,8 antes da autoclavagem à $121^{\circ} \mathrm{C}$ por 20 minutos). O restante das cápsulas foi armazenado por 30 dias à $8^{\circ} \mathrm{C}$ e $15^{\circ} \mathrm{C}$ na ausência de luz e posteriormente inoculadas em meio de regeneração. Após 50 dias de inoculação foram avaliados a porcentagem de ruptura das cápsulas. Para isso foram utilizadas 10 repetições por tratamento.

\subsection{Crescimento lento}

Segmentos nodais com aproximadamente $1 \mathrm{~cm}$ foram excisados de plântulas obtidas a partir da germinação das sementes conforme descrito no item 2.1. Esses explantes foram inoculados em meio MS acrescido com 2,5 $\mu \mathrm{M}$ de BAP com diferentes concentrações de 
sacarose (30, 45 e $\left.60 \mathrm{~g} \mathrm{~L}^{-1}\right)$, acrescido de $7 \mathrm{~g} \mathrm{~L}^{-1}$ de ágar com pH ajustado para 5,8 antes da autoclavagem à $121^{\circ} \mathrm{C}$ por 20 minutos. Após a inoculação o material foi mantido por sete dias em sala de crescimento com temperatura de $25 \pm$ $2{ }^{\circ} \mathrm{C}, 16$ horas de fotoperíodo e posteriormente foi transferido para B.O.D com temperaturas de $8^{\circ} \mathrm{C}$ e $15^{\circ} \mathrm{C}$ na ausência de luz, onde foram mantidos por 90 e 180 dias. Após esses períodos as plântulas foram avaliadas quanto à porcentagem de sobrevivência e então transferidas para meio de regeneração (MS + 5 $\mu \mathrm{M}$ de $\mathrm{BAP}+7 \mathrm{~g} \mathrm{~L}^{-1}$ de ágar, com $\mathrm{pH}$ ajustado para 5,8 antes da autoclavagem à $121^{\circ} \mathrm{C}$ por 20 minutos) e mantidas em fotoperíodo de 16 horas, temperatura de $25 \pm 2^{\circ} \mathrm{C}$ com irradiância de fótons de $36 \mu \mathrm{mol} \mathrm{m} \mathrm{m}^{-2} \mathrm{~s}^{-1}$, onde permaneceram por 45 dias, e então foram avaliadas quanto à porcentagem de regeneração, altura das plantas (cm), número de folhas e de brotos. Para isso foram utilizadas 24 repetições por tratamento, sendo oito frascos com três segmentos em cada.

\subsection{Análises estatísticas}

O delineamento experimental utilizado foi inteiramente casualizado (DIC) com os tratamentos distribuídos em esquema fatorial de 2x3. Para o experimento de armazenamento de unidades encapsuláveis foram consideradas duas concentrações de alginato de sódio (3\% e 4\%) e três concentrações de sacarose $(0,0,25$ e $0,5 \mathrm{M})$, enquanto que para o experimento de crescimento lento foram consideradas duas temperaturas $\left(8^{\circ} \mathrm{C}\right.$ e $\left.15^{\circ} \mathrm{C}\right)$ e três concentrações de sacarose $(30,45$ e $60 \mathrm{~g} \mathrm{~L}-1)$. Os dados obtidos foram analisados comparando-se as médias pelo teste de Scott- knott a 5\% de probabilidade pelo software estatístico Sisvar $^{\circledR}$ (FERREIRA, 2014).

\section{RESULTADOS E DISCUSSÃO}

\subsection{Armazenamento de unidades encapsuláveis}

Após 50 dias de inoculação foi avaliada a porcentagem de ruptura das cápsulas que foram imediatamente inoculadas e daquelas que permaneceram 30 dias armazenadas nas temperaturas de $8^{\circ} \mathrm{C}$ e $15^{\circ} \mathrm{C}$.

Para as unidades encapsuláveis que não passaram pelo processo de armazenamento não foram observadas diferenças na porcentagem de ruptura em nenhuma das concentrações de sacarose utilizadas $(0,0,25$ e $0,5 \mathrm{M})$ para a matriz de alginato de sódio na concentração de 3\%. No entanto, para a matriz de alginato de sódio de $4 \%$ a maior taxa de ruptura (100\%) ocorreu na concentração de $0,5 \mathrm{M}$ de sacarose (Tabela 1). Isso por ser justificado por possível aumento da rigidez da matriz, sendo necessária maior disponibilidade de carboidratos para que as cápsulas tivesse melhor porcentagem de ruptura (SUNDARARAJ, AGRAWAL, TYAGI, 2010 ). No entanto, não foram observadas diferenças na porcentagem de ruptura das cápsulas quando foram comparadas às concentrações de alginato de sódio (3\% e 4\%) (Tabela 1).

Para as unidades encapsuláveis que passaram pelo processo de armazenamento por 30 dias foi possível fazer a conservação apenas na temperatura de $15^{\circ} \mathrm{C}$. A não sobrevivência na temperatura mais baixa de $8^{\circ} \mathrm{C}$ pode ser justificada pelo fato do jenipapo ser uma espécie 
tropical. Confirmando que essas espécies não podem ser armazenadas em temperaturas muito baixas (SUNDARARAJ, AGRAWAL, TYAGI, 2010).

Na temperatura de $15^{\circ} \mathrm{C}$, tanto para as cápsulas de alginato de sódio de $3 \%$ e $4 \%$ os melhores resultados ocorrem quando o prétratamento foi realizado na ausência de sacarose (Tabela 1).

O uso de maiores concentrações para a desidratação osmótico pode ter causado choque osmótico, o que comprometeu o desenvolvimento (SHARMA et al., 2013; SUNDARARAJ, AGRAWAL, TYAGI, 2010).

Além disso, não ocorreram diferenças para a ruptura quando comparada às concentrações de alginato de sódio (Tabela 1). Mostrando que esse aumento da concentração, que poderia causar maior rigidez (SAHOO et al., 2012), não foi suficiente para influenciar de forma negativa na porcentagem de ruptura, o que afirma que é possível fazer a conservação em curto prazo de jenipapo.

Tabela 1 - Porcentagem de ruptura das cápsulas (\%) de alginato de sódio de $3 \%$ e 4\%. Médias seguidas pela mesma letra não diferem entre si pelo teste de Scott-Knott $(\mathrm{p}<0.05)$.

\begin{tabular}{cccc}
\hline \multirow{2}{*}{$\begin{array}{c}\text { Concentração de alginato de } \\
\text { sódio(\%) }\end{array}$} & \multicolumn{3}{c}{ Concentração de sacarose (M) } \\
\cline { 2 - 4 } Sem armazenamento & 0 & 0,25 & 0,5 \\
\hline $3 \%$ & $90 \mathrm{aA}$ & $90 \mathrm{aA}$ & $70 \mathrm{aA}$ \\
$4 \%$ & $60 \mathrm{aB}$ & $60 \mathrm{aB}$ & $100 \mathrm{aA}$ \\
\hline 30 dias de armazenamento a $15^{\circ} \mathrm{C}$ & & & \\
\hline $3 \%$ & $100 \mathrm{aA}$ & $50 \mathrm{aB}$ & $50 \mathrm{aB}$ \\
$4 \%$ & $90 \mathrm{aA}$ & $30 \mathrm{aB}$ & $40 \mathrm{aB}$ \\
\hline
\end{tabular}

* Letras minúsculas comparam os dados entre colunas e letras maiúsculas comparam os dados nas linhas.

\subsection{Crescimento lento}

No intuito de realizar a conservação em médio prazo de jenipapo, utilizou-se a técnica de crescimento lento. Para isso foram realizadas alterações na concentração de sacarose no meio de cultivo e a redução da temperatura de armazenamento.

Pode-se perceber que as brotações de jenipapo podem ser eficientemente conservadas por 90 dias. As plantas armazenadas em $8^{\circ} \mathrm{C}$ apresentaram diferenças na porcentagem de sobrevivência, sendo que a concentração de $45 \mathrm{~g}$ $\mathrm{L}^{-1}$ e $60 \mathrm{~g} \mathrm{~L} \mathrm{~L}^{-1}$ apresentaram os melhores resultados (96\% e 100\%, respectivamente) (Tabela 2), o que provavelmente está relacionado com o fato da sacarose ser uma fonte de carboidratos para esses explantes (TREJGELL, KAMIŃSKA, TRETYN, 2015). Para o armazenamento que foi realizado à $15^{\circ} \mathrm{C}$, a taxa de sobrevivência foi alta (100\%) e não diferiu entre as concentrações de sacarose utilizada. Quando comparada a influência da temperatura de $8^{\circ} \mathrm{C}$ e $15^{\circ} \mathrm{C}$ na porcentagem de sobrevivência, 
pode-se observar diferença apenas quando foi utilizada a concentração de $30 \mathrm{~g} \mathrm{~L}^{-1}$ de sacarose, onde a temperatura de $15^{\circ} \mathrm{C}$ apresentou os melhores resultados (Tabela 2).

Quando a conservação foi prolongada para 180 dias ocorreu declínio na sobrevivência quando comparado com 90 dias de armazenamento. Com 90 dias obteve-se média $94,3 \%$ e $100 \%$ de sobrevivência nas temperaturas de $8^{\circ} \mathrm{C}$ e $15^{\circ} \mathrm{C}$, respectivamente, enquanto que após o armazenamento por 180 dias, a taxa de sobrevivência média foi de 90,3\% e $94,7 \%$, respectivamente. No entanto, mesmo com a redução os resultados ainda foram favoráveis.

Nesse caso, foi possível perceber que as diferentes concentrações de sacarose utilizadas (30 $\mathrm{g} \mathrm{L}^{-1}, 45 \mathrm{~g} \mathrm{~L}^{-1}$ e $60 \mathrm{~g} \mathrm{~L}^{-1}$ ) foram eficientes para fazer a conservação in vitro do jenipapo, não ocorrendo diferenças entre as concentrações (Tabela 2). Esses resultados indicam que uma menor concentração de sacarose é suficiente para manter as taxas de sobrevivência mesmo quando ocorre o retardo do crescimento.

Tabela 2 - Porcentagem de sobrevivência de segmentos nodais de jenipapo após 90 e 180 dias de armazenamento a $8^{\circ} \mathrm{C}$ e $15^{\circ} \mathrm{C}$. Médias seguidas pela mesma letra não diferem entre si pelo teste de Scott-Knott $(\mathrm{p}<0.05)$.

\begin{tabular}{ccc}
\hline \multirow{2}{*}{$\begin{array}{c}\text { Concentração de sacarose } \\
\left(\mathrm{g} \mathrm{L}^{-1}\right)\end{array}$} & \multicolumn{2}{c}{ Sobrevivência (\%) } \\
\cline { 2 - 3 } Após 90 dias & 8 & 15 \\
\hline 30 & $87 \mathrm{bB}$ & $100 \mathrm{aA}$ \\
45 & $96 \mathrm{aA}$ & $100 \mathrm{aA}$ \\
60 & $100 \mathrm{aA}$ & $100 \mathrm{aA}$ \\
\hline Após 180 dias & & \\
\hline 30 & $92 \mathrm{aA}$ & $1 \mathrm{aA}$ \\
45 & $96 \mathrm{aA}$ & $88 \mathrm{aA}$ \\
60 & $83 \mathrm{aA}$ & $96 \mathrm{aA}$ \\
\hline
\end{tabular}

* Letras minúsculas comparam os dados entre colunas e letras maiúsculas comparam os dados nas linhas.

No entanto, avaliar apenas a sobrevivência não é suficiente, visto que, é necessário que a planta retome o crescimento após a etapa de armazenamento. Dessa forma, a etapa de regeneração dos explantes é crítica durante o desenvolvimento de um protocolo de crescimento lento, sendo necessário avaliar a porcentagem de explantes que foram regenerados. Tanto após 90 e 180 dias de armazenamento não foi observada retomada do crescimento das plantas armazenadas à $8^{\circ} \mathrm{C}$ em todas as concentrações de sacarose avaliadas.
Isso pode ser devido ao estresse imposto aos segmentos nodais quando eles foram transferidos das condições de cultivo de armazenamento a frio para as condições ótimas, sendo que nesse caso a baixa temperatura pode ter reduzido fortemente a atividade metabólica das plantas não permitindo a retomada do crescimento (OZUDOGRU et al., 2013). Além disso, essa não retomada de crescimento de plantas armazenadas à $8^{\circ} \mathrm{C}$ pode ser devido ao fato do jenipapo ser uma planta tropical, sendo que essas temperaturas mais baixas geralmente são 
indicadas para espécies temperadas, assim é necessário que a espécie seja armazenada em temperaturas mais elevadas, o que justifica os melhores resultados pós-conservação encontrados para as plantas armazenadas a $15^{\circ} \mathrm{C}$. Esses resultados são semelhantes aos encontrados para o armazenamento de unidades encapsuláveis, comprovando que a espécie não deve ser armazenada em temperaturas de $8^{\circ} \mathrm{C}$.

No desenvolvimento pós-conservação, após 90 dias os melhores resultados de regeneração à $15^{\circ} \mathrm{C}$ foram encontrados com $45 \mathrm{~g}$ $\mathrm{L}^{-1}$ e $60 \mathrm{~g} \mathrm{~L} \mathrm{~L}^{-1}$ de sacarose $(88$ e $96 \%$, respectivamente). Para a altura das plantas não foram observadas diferenças, no entanto, o número de brotações e de folhas foram maiores com $45 \mathrm{~g} \mathrm{~L}^{-1}$ de sacarose (Tabela 3). Já após 180 dias, a concentração de $60 \mathrm{~g} \mathrm{~L}^{-1}$ proporcionou maior porcentagem de regeneração $(67 \%)$ das plantas sobreviventes, bem como maior número de brotos e de folhas (Tabela 3). Esses resultados podem estar relacionados com o fato de uma concentração maior de sacarose durante o armazenamento ter proporcionado aumento de reserva de carboidratos nos tecidos, o que pode acarretar em aumento do potencial de regeneração, uma vez que, a sacarose é uma das principais fontes de carbono prontamente disponível (SANTOS, et al., 2011; TREJGELL, KAMIŃSKA, TRETYN, 2015).

Tabela 3 - Avaliação 45 dias após a inoculação dos explantes em meio de regeneração. Médias seguidas pela mesma letra não diferem entre si pelo teste de Scott-Knott $(\mathrm{p}<0.05)$.

\begin{tabular}{ccccc}
\hline $\begin{array}{c}\text { Concentração } \\
\text { de sacarose } \\
\left(\mathrm{g} \mathrm{L}^{-1}\right)\end{array}$ & Regeneração (\%) & Altura $(\mathrm{cm})$ & Número de folhas & Número de brotações \\
\hline 90 dias & $63 \mathrm{~b}$ & $1.96 \mathrm{a}$ & $3.25 \mathrm{~b}$ & $1.45 \mathrm{~b}$ \\
\hline 30 & $88 \mathrm{a}$ & $2.22 \mathrm{a}$ & $5.63 \mathrm{a}$ & $2.08 \mathrm{a}$ \\
45 & $96 \mathrm{a}$ & $2.15 \mathrm{a}$ & $3.25 \mathrm{~b}$ & $1.67 \mathrm{~b}$ \\
\hline 60 & & & $0.37 \mathrm{~b}$ & $1.17 \mathrm{~b}$ \\
\hline 180 dias & $29 \mathrm{~b}$ & $1.97 \mathrm{a}$ & $0.96 \mathrm{~b}$ & $1.08 \mathrm{~b}$ \\
30 & $50 \mathrm{a}$ & $2.09 \mathrm{a}$ & $2.67 \mathrm{a}$ & $1.46 \mathrm{a}$ \\
\hline
\end{tabular}

\section{CONSIDERAÇÕES FINAIS}

Pode-se concluir que para fazer a conservação em curto prazo de jenipapo deve-se utilizar a matriz de alginato de sódio a $3 \%$ com um pré-tratamento sem a presença de sacarose. Já para a conservação em médio prazo, é possível utilizar a técnica de crescimento lento por até 180 dias. Para isso o material deve ser armazenado a $15^{\circ} \mathrm{C}$ com acréscimo de $60 \mathrm{~g} \mathrm{~L}^{-1} \mathrm{de}$ sacarose ao meio de cultura. 


\section{AGRADECIMENTOS}

À Universidade Federal de Lavras (UFLA), CAPES, CNPq e FAPEMIG.

\section{REFERÊNCIAS}

ALMEIDA, C. S. et al. Respostas morfogenéticas de jenipapeiro em diferentes condições de cultura in vitro. Revista Caatinga, v. 28, n. $1,2015$.

BRASIL. INSTITUTO BRASILEIRO DE GEOGRAFIA E ESTATÍSTICA - IBGE. Brasil em síntese: território. 2004. Disponível em: <https://brasilemsintese.ibge.gov.br/territorio.ht m>. Acesso em: 25 maio 2018.

DAUD, N.; TAHA, R. M.; HASBULLAH, N. A. Artificial seed production from encapsulated micro shoots of Saintpaulia ionantha Wendl.(African Violet). Journal of Applied Science, v. 8, n. 24, p. 4662-4667, 2008.

ENGELMANN, F. Use of biotechnologies for the conservation of plant biodiversity. In Vitro Cellular \& Developmental Biology-Plant, v. 47, n. 1, p. 5-16, 2011.

FERREIRA, D. F.. Sisvar: a Guide for its Bootstrap procedures in multiple comparisons.Ciência e Agrotecnologia, v. 38, n. 2, p. 109-112, 2014.

FERNANDES, L. A. et al. Crescimento inicial, níveis críticos de fósforo e frações fosfatadas em espécies florestais.Pesquisa Agropecuária

Brasileira, v. 35, n. 6, p. 1191-1198, 2000.

LAMBARDI, M., OZUDOGRU E. A. Advances in the safe storage of micropropagated woody plants at low temperature. Acta Horticulture, v. 988, p 29-42, 2012.

LÉDO, ANA SILVA et al. Medium-term conservation and regrowth of the tropical multipurpose species Genipa americana. African Journal of Agricultural Research, v. 10, n. 41, p. 3885-3892, 2015.

MURASHIGE, T.; SKOOG, F.. A revised medium for rapid growth and bio assays with tobacco tissue cultures. Physiologia plantarum, v. 15 , n. 3, p. 473-497, 1962.
OZUDOGRU, E. A.; PREVIATI, A.;

LAMBARDI, $M$. In vitro conservation and cryopreservation of ornamental plants. In: Protocols for in vitro propagation of ornamental plants. Humana Press, 2010. p. 303-324.

OZUDOGRU, E.A. et al. In vitro conservation and cryopreservation of Nandina domestica, an outdoor ornamental shrub. Notulae Botanicae Horti Agrobotanici Cluj-Napoca, v.41, p.638645, 2013

RAI, M. K.; JAISWAL, V. S.; JAISWAL, U. Encapsulation of shoot tips of guava (Psidium guajava L.) for short-term storage and germplasm exchange. Scientia Horticulturae, v. 118, n. 1, p. 33-38, 2008.

SÁ, F. P. et al. Encapsulamento, crioproteção e desidratação na capacidade regenerativa de ápices caulinares de Genipa americana. Ciência Rural, v. 45, n. 11, p. 1939-1945, 2015.

SANTANA, K. B. et al. Physiological analyses of Genipa americana L. reveals a tree with ability as phytostabilizer and rhizofilterer of chromium ions for phytoremediation of polluted watersheds. Environmental and experimental botany, v. 80, p. 35-42, 2012.

SANTOS, M. C. et al. Efeito da sacarose e do sorbitol na conservação in vitro de segmentos nodais de mangabeira. Revista Ciência Agronômica, v. 42, n. 3, p. 735-741, 2011.

SHARMA, S. et al. Synseed technology-a complete synthesis. Biotechnology advances, v. 31, n. 2, p. 186-207, 2013.

SILVA, D. P. C. S. et al. In vitro conservation of ornamental plants. Ornamental Horticulture. v. 24, n . 1, p. 28-33, 2018.

SOUZA, R. R. et al. Optimization of jenipapo in vitro seed germination process. Ciência e Agrotecnologia, v. 40, n. 6, p. 658-664, 2016.

STRASSBURG, B. B. N. et al. Moment of truth for the Cerrado hotspot. Nature, Ecology \& Evolution, v. 1, p. 1-3, 2017. 
SUNDARARAJ, S. G.; AGRAWAL, A.;

TYAGI, R. K. Encapsulation for in vitro shortterm storage and exchange of ginger (Zingiber officinale Rosc.) germplasm. Scientia

Horticulturae, v. 125, n. 4, p. 761-766, 2010.

TREJGELL, A.; KAMIŃSKA, M.; TRETYN, A.. In vitro slow growth storage of Senecio macrophyllus shoots. Acta Physiologiae

Plantarum, v. 37, n. 11, p. 234, 2015.

Junia Rafael Mendonça Figueiredo

Doutora em Fisiologia Vegetal pela Universidade Federal de Lavras (UFLA). Docente do curso de Agronomia da Universidade Vale do Rio Verde (UninCor)

\section{Patrícia Duarte de Oliveira Paiva}

Doutora em Fitotecnia pela Universidade Federal de Lavras (UFLA). Professora titular da Universidade Federal de Lavras (UFLA).

\section{Renato Paiva}

Doutor em Agronomia pela University of Illinois at Urbana-Champaign. Professor titular da Univeridade Federal de Lavras (UFLA).

\section{Diogo Pedrosa Corrêa da Silva}

Doutor em Fisiologia Vegetal pela Universidade Federal de Lavras (UFLA). Pós-doutorando do programa de pós-graduação em Fitotecnia da Universidade Federal de Lavras (UFLA)

\section{Raquel Mesquita}

Graduanda em Ciência Biológicas pela Universidade Federal de Lavras (UFLA).

\section{Camila Vitória Nunes de Faria}

Doutora em Fisiologia Vegetal pela Universidade Federal de Lavras (UFLA).

\footnotetext{
Michele Valquíria dos Reis

Doutora em Fisiologia Vegetal pela Universidade Federal de Lavras (UFLA). Pós-doutoranda do programa de pós-graduação em Fisiologia Vegetal da Universidade Federal de Lavras (UFLA)
} 